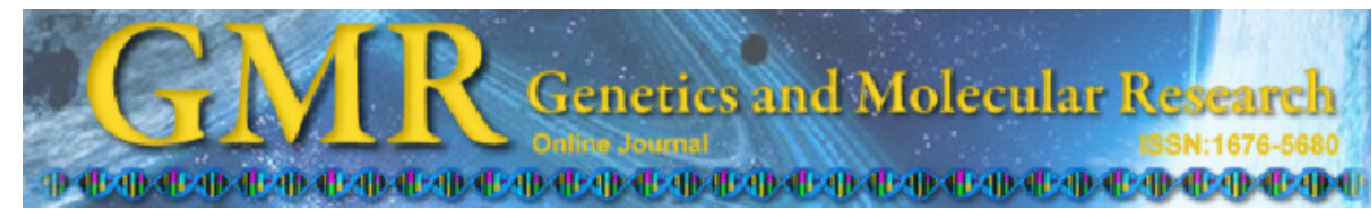

\title{
Demographic characterization of Brazilian patients enrolled in the Fabry Registry
}

\author{
A.M. Martins ${ }^{1}$, S.O. Kyosen ${ }^{1}$, J. Garrote ${ }^{1}$, F.M.V. Marques ${ }^{2}$, \\ J.G. Guilhem ${ }^{3}$, E. Macedo ${ }^{4}$, J. Sobral Neto ${ }^{5}$ and S. Ura ${ }^{6}$ \\ ${ }^{1}$ Centro de Referência em Erros Inatos do Metabolismo, \\ Departamento de Pediatria, Universidade Federal de São Paulo, \\ São Paulo, SP, Brasil \\ ${ }^{2}$ Hospital Santo Antonio, Tapejara, RS, Brasil \\ ${ }^{3}$ Instituto de Hemodiálise Sorocaba, Sorocaba, SP, Brasil \\ ${ }^{4}$ Centro de Infusão de Doenças Raras, Curimatá, PI, Brasil \\ ${ }^{5}$ Centrocard, Centro de Avaliação Cardiológica, Brasília, DF, Brasil \\ ${ }^{6}$ Instituto Lauro de Souza Lima, Bauru, SP, Brasil \\ Corresponding author: S.O. Kyosen \\ E-mail: skyosen.dped@epm.br / skyosen@gmail.com
}

Genet. Mol. Res. 12 (1): 136-142 (2013)

Received June 21, 2012

Accepted November 2, 2012

Published January 24, 2013

DOI http://dx.doi.org/10.4238/2013.January.24.5

\begin{abstract}
Fabry disease (FD) is an X-linked inborn error of metabolism caused by alpha-galactosidase A deficiency. The Fabry Registry is an ongoing observational database that compiles clinical data on patients with FD. We analyzed the Fabry Registry data of patients enrolled in Brazil to characterize the demographic and baseline clinical characteristics of this patient population. As of October 2010, 126 Brazilian patients were enrolled in the Registry (61 males, 65 females). The median age at onset of symptoms in males was 9.8 years, compared to 11.4 years in females. Males were diagnosed at a median age of 31.9 years and females at 27.1 years. The median time between the onset of first symptoms and diagnosis was 20.3 years in males and 14.3 years in females. Neurologic pain was the presenting symptom most frequently reported by both genders. Renal events were the most common clinical events reported in males, while cardiac events were the most common events in females. The results of
\end{abstract}


these analyses indicate that Brazilian patients were frequently not diagnosed with FD until many years after the onset of symptoms. Many Brazilian Fabry Registry patients report experiencing neurological pain, and many Brazilian women with FD exhibit substantial signs and symptoms. The prevalence of neurological pain as a presenting symptom among Brazilian Registry patients is consistent with previous reports from the overall Registry population. FD is treatable, and earlier diagnosis will allow for prompt initiation of appropriate treatment that may avert irreversible damage that could occur during the time from symptom onset to diagnosis.

Key words: Fabry disease; Alpha-galactosidase A deficiency; Fabry Registry; Brazil; Demographics

\section{INTRODUCTION}

Fabry disease (FD) is an X-linked inborn error of metabolism caused by a deficiency of the lysosomal enzyme alpha-galactosidase A (Desnick et al., 2001). The disease process starts in infancy, although the majority of patients remain clinically asymptomatic during the very first years of life. The first clinical symptoms, including neuropathic pain in the extremities, hypohidrosis, and gastrointestinal symptoms such as abdominal pain, diarrhea, and food intolerance, typically appear between the ages of 3 and 10 years, with presentation in girls being reported a few years later than that in boys (Hopkin et al., 2008; Wilcox et al., 2008). As individuals with FD age, the accumulation of globotriaosylceramide results in progressive damage to vital organ systems, organ failure, and subsequently, a shortened life expectancy due to end-stage renal disease and life-threatening cardiovascular or cerebrovascular complications (Branton et al., 2002; Mehta et al., 2004; Schiffmann et al., 2009; Sims et al., 2009). FD has long been regarded as an adult disease primarily affecting males, while it was believed that female patients were simply carriers who were protected against development of the disease symptoms and events. However, evolving knowledge about the natural history of the disease suggests that a high percentage of female patients develop vital organ involvement, including that of the kidneys, heart, and/or brain, albeit approximately a decade later than males (Wilcox et al., 2008).

There are many variations in the clinical manifestations of FD among individual patients regardless of gender, and there may also be important differences in the clinical manifestations of patients residing in different regions of the world. In the present report, we analyzed Fabry Registry data from patients enrolled in Brazil to characterize the demographic and baseline clinical characteristics of this patient population. The Fabry Registry (www.fabryregistry. com) is an international observational voluntary program initiated in 2001. It is a global outcomes assessment and disease management program that compiles patient outcome data from routine clinical practice to provide the medical community with resources to help optimize patient care. The primary objectives of the Fabry Registry are to enhance understanding of the variability, progression, and natural history of FD, including of heterozygous females with the disease; to assist the Fabry medical community with the development of recommendations for monitoring patients and reports on patient outcomes to help optimize patient care; to characterize and describe the Fabry population as a whole, and to evaluate the long-term safety and effectiveness of treatment. 


\section{MATERIAL AND METHODS}

The Fabry Registry is an ongoing observational database that compiles clinical and investigative data on patients with FD. It is sponsored and administered by Genzyme, a Sanofi company (Cambridge, MA, USA). The Fabry Registry is the largest global assessment program designed to collect clinical data related to the onset and progression of FD. All FD patients are eligible to enroll in the Registry regardless of symptoms or reception of enzyme replacement therapy (ERT). Patient and physician participation is voluntary. All patients provide informed consent through a process approved by local Institutional Review Boards/Ethics Committees.

Treating physicians determine the actual frequency of assessments according to patients' individualized needs, although the Fabry Registry provides participating physicians with a recommended schedule of assessments. Given the voluntary nature of the data reporting, patients' ages at clinical assessments and time intervals between assessments are variable. Data collected by physicians or their designees are submitted to the Fabry Registry for central processing through a set of standardized electronic case report forms.

In the present analyses, we characterized Brazilian Fabry Registry patients according to baseline demographics and the occurrence of clinical events. Only natural history data (i.e., obtained from untreated patients receiving ERT or prior to treatment with ERT) were included. Patients for whom ERT status was unknown were excluded from the analyses to ensure that clinical events occurred prior to initiation of ERT. Significant clinical events were categorized as cardiovascular, cerebrovascular, or renal. Cardiovascular events were defined as myocardial infarction, congestive heart failure, arrhythmia, angina pectoris, or significant cardiac procedures (including pacemaker implantation, coronary bypass, implantable cardioverter defibrillator, stent placement, valve replacement, or balloon pump). Cerebrovascular events were defined as strokes. Renal events were defined as chronic renal dialysis ( $\geq 40$ days) or renal transplantation.

Analyses were summarized using descriptive statistics. Mean, standard deviation, median, minimum, and maximum values were calculated for continuous variables, and percentages were calculated for categorical variables. All data analyses were performed using the SAS statistical software system version 9.2 (USA).

\section{RESULTS}

As of October 2010, 126 Brazilian patients with known ERT status were enrolled in the Fabry Registry (61 males, 65 females). Demographic data are presented in Table 1. The majority of the Brazilian patients in the Fabry Registry were Caucasian; 5.7\% were Afro-Brazilian. The median age at onset of symptoms in females was 11.4 years, compared to 9.8 years in males, while the mean age of symptom onset was 13.8 years and 13.4 years in females and males, respectively. In both males and females, the median age at diagnosis was much later than the age at onset of symptoms, with males being diagnosed at a median age of 31.9 years and females at 27.1 years. The median time between the onset of first symptoms and diagnosis was 20.3 years in males and 14.3 years in females, and the mean time from symptom onset to diagnosis for males and females was similar (20.1 and 19.1 years, respectively). 
Table 1. Demographic characteristics of Brazilian Fabry Registry patients.

\begin{tabular}{|c|c|c|}
\hline & \multicolumn{2}{|c|}{ Total No. of patients enrolled } \\
\hline & Males (61) & Females (65) \\
\hline \multicolumn{3}{|l|}{ Ethnicity } \\
\hline Caucasian & $27(44.3)$ & $41(63.1)$ \\
\hline Black & $4(6.6)$ & $2(3.1)$ \\
\hline Hispanic & $9(14.8)$ & $14(21.5)$ \\
\hline Other & $18(29.5)$ & $7(10.8)$ \\
\hline Unknown/missing & $3(4.9)$ & $1(1.5)$ \\
\hline \multicolumn{3}{|c|}{ Age as of October 2010 (years) } \\
\hline $\mathrm{N}$ & 61 & 65 \\
\hline Mean (SD) & $39.4(14.70)$ & $36.5(16.10)$ \\
\hline Median (min, max) & $38.1(9.1,77.6)$ & $34.4(8.9,74.3)$ \\
\hline \multicolumn{3}{|l|}{ Age category (years) } \\
\hline $0-<10$ & $1(1.6)$ & $1(1.5)$ \\
\hline$\geq 10-<20$ & $6(9.8)$ & $11(16.9)$ \\
\hline$\geq 20-<30$ & $7(11.5)$ & $16(24.6)$ \\
\hline$\geq 30-<40$ & $18(29.5)$ & $11(16.9)$ \\
\hline$\geq 40-<50$ & $14(23.0)$ & $9(13.8)$ \\
\hline$\geq 50$ & $15(24.6)$ & $17(26.2)$ \\
\hline \multicolumn{3}{|c|}{ Age at symptom onset (years) } \\
\hline $\mathrm{N}$ & 55 & 49 \\
\hline Mean (SD) & $13.4(11.50)$ & $13.8(9.47)$ \\
\hline Median (min, max) & $9.8(0.8,61.5)$ & $11.4(4.1,49.2)$ \\
\hline \multicolumn{3}{|l|}{ Age category (years) } \\
\hline $0-<10$ & $29(47.5)$ & $20(30.8)$ \\
\hline$\geq 10-<20$ & $17(27.9)$ & $20(30.8)$ \\
\hline$\geq 20-<30$ & $5(8.2)$ & $6(9.2)$ \\
\hline$\geq 30-<40$ & 0 & $1(1.5)$ \\
\hline$\geq 40-<50$ & $3(4.9)$ & $2(3.1)$ \\
\hline$\geq 50$ & $1(1.6)$ & 0 \\
\hline \multicolumn{3}{|l|}{ Age at diagnosis (years) } \\
\hline $\mathrm{N}$ & 56 & 58 \\
\hline Mean (SD) & $31.5(14.11)$ & $30.5(15.80)$ \\
\hline Median (min, max) & $31.9(2.2,62.3)$ & $27.1(5.0,72.6)$ \\
\hline \multicolumn{3}{|l|}{ Age category (years) } \\
\hline $0-<10$ & $3(4.9)$ & $4(6.2)$ \\
\hline$\geq 10-<20$ & $8(13.1)$ & $14(21.5)$ \\
\hline$\geq 20-<30$ & $15(24.6)$ & $15(23.1)$ \\
\hline$\geq 30-<40$ & $15(24.6)$ & $7(10.8)$ \\
\hline$\geq 40-<50$ & $9(14.8)$ & $11(16.9)$ \\
\hline$\geq 50$ & $6(9.8)$ & $7(10.8)$ \\
\hline \multicolumn{3}{|c|}{ Time from symptom onset to diagnosis (years) } \\
\hline $\mathrm{N}$ & 48 & 44 \\
\hline Mean (SD) & $20.1(13.59)$ & $19.1(14.84)$ \\
\hline Median (min, max) & $20.3(0.0,50)$ & $14.3(0.0,65)$ \\
\hline
\end{tabular}

$\mathrm{SD}=$ standard deviation; $\min =$ minimum; $\max =$ maximum.

A summary of Fabry symptom onset by organ system among male and female patients is provided in Figure 1. Neurologic pain was the presenting symptom most frequently reported by both genders ( $80 \%$ of males and $62 \%$ of females), followed by dermatological findings, which were reported in approximately $14 \%$ of males and $3 \%$ of females.

The age at which Brazilian patients in the Registry first reported a clinical event is presented in Table 2. Male patients were more likely to report a clinical event compared to females. Renal events were the most common clinical events reported in males (17 patients), first occurring at a median age of 38.0 years, while cardiac events were the most common events in females (6 patients), occurring at a median age of 44.2 years. 


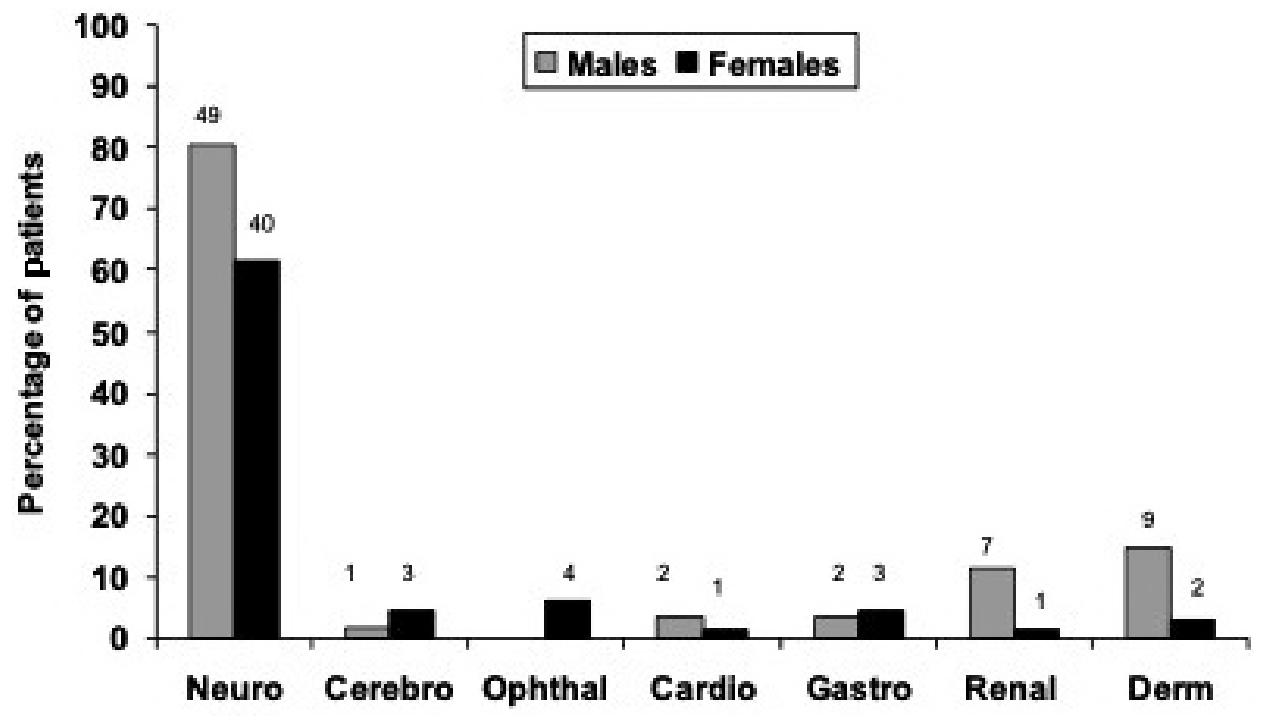

Figure 1. Summary of presenting symptoms at enrollment by organ system. Data are expressed as percentages of patients who reported the indicated symptoms at the time of enrollment. Numbers above the bars indicate the number of patients in each category. Neuro = neurologic pain; Cerebro = cerebrovascular; Ophthal = ophthalmological; Cardio $=$ cardiovascular; Gastro $=$ gastrointestinal; Derm $=$ dermatological.

Table 2. Summary of clinical events (renal, cardiovascular, or cerebrovascular) and age at first clinical event during natural history period.

\begin{tabular}{|c|c|c|}
\hline & \multicolumn{2}{|c|}{ Total No. of patients with a clinical event during the period } \\
\hline & Males (61) & Females (65) \\
\hline \multicolumn{3}{|c|}{ Patients reporting renal event during natural history period } \\
\hline \multicolumn{3}{|c|}{ Age at first renal event (years) } \\
\hline $\mathrm{N}$ & 17 & 1 \\
\hline Mean (SD) & $37.9(12.02)$ & $37.4(-)$ \\
\hline Median (range) & $38.0(19.2,66.4)$ & $37.4(37.4,37.4)$ \\
\hline \multirow{2}{*}{\multicolumn{3}{|c|}{$\begin{array}{l}\text { Patients reporting cardiovascular event during natural history period } \\
\text { Age at first cardiovascular event (years) }\end{array}$}} \\
\hline \multicolumn{2}{|c|}{ Age at first cardiovascular event (years) } & \\
\hline $\mathrm{N}$ & 9 & 6 \\
\hline Mean (SD) & $46.9(14.88)$ & $42.7(10.04)$ \\
\hline Median (range) & $44.2(19.8,72.6)$ & $44.2(24.2,54.1)$ \\
\hline \multicolumn{3}{|c|}{ Patients reporting cerebrovascular event during natural history period } \\
\hline \multicolumn{3}{|c|}{ Age at first cerebrovascular event (years) } \\
\hline $\mathrm{N}$ & 6 & 3 \\
\hline Mean (SD) & $44.4(14.79)$ & $40.1(8.42)$ \\
\hline Median (range) & $40.4(32.0,73.6)$ & $42.3(30.8,47.2)$ \\
\hline
\end{tabular}

$\mathrm{SD}=$ standard deviation

\section{DISCUSSION}

We analyzed the Fabry Registry data of patients enrolled in Brazil to characterize the demographic and baseline clinical characteristics of this patient population. In general, it ap- 
pears that FD is less prevalent in the Afro-Brazilian population compared to other ethnicities. Wilcox et al. (2008) previously reported that $1.5 \%$ of the overall Fabry Registry population was Afro-Brazilian, and in the present analyses, only 5.7\% (6 of 126 patients) of Brazilian Registry patients was Afro-Brazilian, despite the fact that in Brazil, $50.6 \%$ of the population is Afro-Brazilian (www.brasil.gov.br/sobre/geografia/populacao/etnias).

The results of the present analyses indicate that Brazilian patients were frequently not diagnosed with FD until many years after the onset of symptoms. The median time between the onset of symptoms and diagnosis in this population was 20.3 years for males and 14.3 years for females, which is longer than that previously reported for the overall Fabry Registry population (11 years for males and 11.4 years for females) (Wilcox et al., 2008). Eighty percent of Brazilian males and $63 \%$ of Brazilian females reported neurological pain as the presenting symptom, which is higher than the rates reported for the overall Registry population $(63 \%$ of males and $43 \%$ of females) (Wilcox et al., 2008). It is possible that patients with more severe disease tend to visit their doctors and have their assessments reported more often than patients with milder disease do. Further investigations will be needed to determine whether FD severity in Brazilian patients differs compared to patients in the rest of the world. In addition, an important consideration in interpreting the data presented in this study is the voluntary and observational nature of the Fabry Registry; therefore, it may also be that the Brazilian sites are more likely to assess and report neurological pain data than the general Registry population. As this is our first report of Brazilian demographic data, more studies on FD in this population are needed to confirm these hypotheses.

Cardiac events were the most common type of event in Brazilian women. Renal events were the most common type of clinical event in Brazilian men, which differs from what is observed in the Fabry Registry as a whole, and is likely due to the small sample size of Brazilian male patients. Overall, cardiac events are the most common type of event reported in Fabry Registry men and women (Eng et al., 2007; Wilcox et al., 2008).

Physicians are encouraged to collaborate, share observations, and generate hypotheses for evaluation, as well as assist in the collection of clinical data for the Fabry Registry in an effort to guide and assess future therapeutic interventions. A better understanding of the natural history of clinical manifestations of FD via the Registry may provide valuable information about which patients may be at greatest risk. Such information can raise awareness of FD among the Brazilian medical community and highlight the importance of improved monitoring and management options.

FD is treatable, and earlier diagnosis will allow for prompt initiation of appropriate treatment. In turn, this may avert irreversible organ damage that could develop during the time between symptom onset and diagnosis. The Brazilian medical community should be aware of the natural history of FD in order to establish the diagnosis during the early stages of disease progression, before the development of severe renal and/or cardiac complications.

\section{ACKNOWLEDGMENTS}

The authors acknowledge the participation of physicians around the world who contributed data to the Registry, their clinic staff, and especially, the patients. We also acknowledge our colleagues at Genzyme, a Sanofi company (Cambridge, MA, USA), for assistance with manuscript writing (Laura Artiles-Carloni) and statistical analyses (Roberta Lemay). 


\section{Disclosures}

The Fabry Registry is sponsored by Genzyme and A.M. Martins is a member of the Fabry Registry Board of Advisors.

\section{REFERENCES}

Anonymous (2011). Available at [www.brasil.gov.br/sobre/geografia/populacao/etnias]. Accessed October 19, 2011.

Branton MH, Schiffmann R, Sabnis SG, Murray GJ, et al. (2002). Natural history of Fabry renal disease: influence of alpha-galactosidase A activity and genetic mutations on clinical course. Medicine 81: 122-138.

Desnick RJ, Ioannou YA and Eng CM (2001). Alpha-Galactosidase A Deficiency: Fabry Disease. In: The Metabolic Bases of Inherited Disease (Scriver C, Beaudet A, Sly W and Valle D, eds.). 8th edn. McGraw-Hill, New York, 3733-3774.

Eng CM, Fletcher J, Wilcox WR, Waldek S, et al. (2007). Fabry disease: baseline medical characteristics of a cohort of 1765 males and females in the Fabry Registry. J. Inherit. Metab. Dis. 30: 184-192.

Hopkin RJ, Bissler J, Banikazemi M, Clarke L, et al. (2008). Characterization of Fabry disease in 352 pediatric patients in the Fabry Registry. Pediatr. Res. 64: 550-555.

Mehta A, Ricci R, Widmer U, Dehout F, et al. (2004). Fabry disease defined: baseline clinical manifestations of 366 patients in the Fabry Outcome Survey. Eur. J. Clin. Invest. 34: 236-242.

Schiffmann R, Warnock DG, Banikazemi M, Bultas J, et al. (2009). Fabry disease: progression of nephropathy, and prevalence of cardiac and cerebrovascular events before enzyme replacement therapy. Nephrol. Dial. Transplant. 24: 2102-2111.

Sims K, Politei J, Banikazemi M and Lee P (2009). Stroke in Fabry disease frequently occurs before diagnosis and in the absence of other clinical events: natural history data from the Fabry Registry. Stroke 40: 788-794.

Wilcox WR, Oliveira JP, Hopkin RJ, Ortiz A, et al. (2008). Females with Fabry disease frequently have major organ involvement: lessons from the Fabry Registry. Mol. Genet. Metab. 93: 112-128. 